\title{
New Health Information Systems (HIS) Quality-in-Use Model Based on the GQM Approach and HCI Principles
}

\author{
Reem Al-Nanih ${ }^{1}$, Hana Al-Nuaim ${ }^{1}$, and Olga Ormandjieva ${ }^{2}$ \\ ${ }^{1}$ Department of Computer Science, King Abdul Aziz University, Saudi Arabia \\ ${ }^{2}$ Dept of Computer Science \& Software Engineering, Concordia University, Canada \\ reem_n2@yahoo.com, hnuaim@kau.edu.sa, ormandj@cse.concordia.ca
}

\begin{abstract}
Human Computer Interaction (HCI) is concerned with the design, evaluation, and implementation of interactive computing systems for human use. HCI is important in Health Information Systems (HIS), because misunderstandings arising because of poorly designed interfaces may lead to medical errors. This paper proposes a Quality-in-Use Model for HIS user interfaces, which identifies HIS-specific quality-in-use goals based on HCI principles, such as Mental Model, Metaphor, Visibility, Affordance, and Feedback. The Goal Question Metric (GQM) method was applied to build the new quality-inuse model applicable to most HIS systems. The resulting quality model is tailored for use in the medical field and reflects the values and viewpoints of the various user groups affected (e.g. doctors and nurses). Its qualitative and quantitative feedback can play a constructive and instructive role in medical institutions such as hospitals, and improve user productivity, satisfaction, and performance.
\end{abstract}

Keywords: Health Information System (HIS), Human Computer Interaction (HCI), Goal Question Metrics (GQM), Quality-in-Use.

\section{Introduction}

In the medical field, the reality is that medical errors are seldom a result of carelessness or negligence. More commonly, they are caused by faulty system design specifically faulty interface design. The goal of this paper is to improve the quality of Health Information Systems (HIS) interface designs by applying the goal-driven approach to defining quality from the Human Computer Interaction (HCI) perspective, which is concerned with the design, evaluation, and implementation of interactive computing systems for human use. The Goal Question Metric (GQM) method [1] was applied to build a new quality-in-use model applicable to most HIS systems, such that: i) the quality model would be tailored to the medical field and its specific user goals; ii) quality feedback would play a constructive and instructive role in medical institutions such as hospitals; iii) the quality characteristics would reflect the values and viewpoints of the various user groups affected (e.g. doctors and nurses); and iv) the quality model would be based on HCI principles such as Mental Model, Metaphor, Visibility, Affordance, and Feedback. The quality-in-use goals selected were inspired by the ISO/IEC 9126-4 Quality-in-Use Standard [14], and aim to increase HIS users' performance, productivity, 
and satisfaction. The proposed quality-in-use model was evaluated on the Phoenix Health Information System (PHIS) currently in use at the King Abdulaziz University Hospital (KAUH). The application of our HIS Quality-in-Use model to the PHIS and the analysis of the quality feedback helped us produce a system (PHIS2) designed to minimize human error, reduce user frustration, and help doctors - with little or no training - work in a more pleasant, efficient, and effective way. The rest of the paper is organized as follows: Related work on HIS evaluation and HIS quality modeling is reviewed in section 2. Section 3 introduces the HCI principles required to understand the quality-in-use model proposed in this paper. The model itself is described in section 4. The results of the empirical validation on a case study are summarized in section 5 . The conclusions and directions for future work are outlined in section 6 .

\section{Motivation and Related Work}

Computers are an integral part of modern medicine and diagnosis. This is especially so in hospitals, as they constitute the core of all advanced medical investigation methods such as ultrasound, computerized tomography, and magnetic resonance imaging [2]. HIS users need interfaces tailored to their specific requirements to facilitate their work and to help them avoid misunderstandings which may lead to medical errors. This need is still underappreciated by software designers [2]. The above motivated the research reported in this paper. HCI and human factors have a significant role to play in increasing the quality of HIS, and consequently in reducing the number of errors, especially those associated with the use of medical devices [5]. Finding design problems in an existing system related to human factors can be a challenge, however, because flaws in user interfaces can be subtle [6].

To the best of the authors' knowledge, very little has been published on the quality modeling of HIS user interfaces. There has been a study of a hospital's order-entry system which identified twenty-two ways in which the system caused patients to be administered the wrong medicine [7]. Most of the issues involved were related to usability problems; for example, at times, users had to review up to twenty screens to see all of a patient's medications. In [3], the role of evaluation in the design of HIS is emphasized, and a framework for considering evaluation methods ranging from controlled experimental approaches to naturalistic observational approaches applicable throughout a system's entire development life cycle is proposed. The problem of defining a quality model for evaluating HIS design is addressed in [4]. The model, based on the ISO/IEC 9126 external and internal product design quality, has been interpreted to meet the requirements of some classes of typical HCI system applications, and exploits experience gained both in the field of medical informatics and in the assessment of software products. The values that result from weighing the quality characteristics according to evidence of their criticality constitute a quality profile which can be used both for evaluation and certification of HIS design and implementation from developers' point of view.

The research reported in this paper differs considerably from the related work in the view on quality of HIS user interfaces. Our objective is to build the HIS Qualityin-Use model from the user's view in terms of HCI principles, which encompass the richness in HIS functionality and represent a broader view than software usability. 


\section{HCI Principles}

$\mathrm{HCI}$ is the study of the interaction between users and computers that occurs through the user interface [9]. It is a discipline which deals not only with the design of the screens and menus, but also with the reasoning underlying the building of functionality into the system in the first place [10]. To be able to design and implement an effective interface, the following principles are fundamental [9]:

Mental Models. The designer must keep in mind the knowledge that a user may have from his or her experience in the real world and how this may be applied to the interface. HCI practitioners concentrate on the definition of mental models as a set of beliefs about how a system works. People interact with systems based on these beliefs [11]. The main problem with mental models is that sometimes humans make incorrect assumptions about the new concepts they encounter, which leads to incorrect use of the system, which in turn slows and hinders the user's progress [10]. In addition to this, mental models tend to be incomplete, because they are simpler than the entities they represent [10].

Metaphors. Some characteristics of users' mental models are metaphors [8; 10]. Metaphors provide short-cuts to understanding difficult concepts, and they can be used to shape user behavior in circumstances that are unfamiliar and that they might otherwise find confusing [10]. For example, the Recycle Bin in MicroSoft Windows@ is the counterpart of the garbage can that people are accustomed to seeing in the real world $[8 ; 12]$.

Visibility. According to the principle of visibility, the user interface should always help the user understand the current state of the system and the operations that can be performed [13]. For example, when you position the cursor over a point on the screen, it should be clear what would happen if you clicked the mouse.

Affordance. Perceived affordance is the quality that makes it easy for a user to spot and identify the functionalities that an interface offers [12].

Feedback. Feedback is information that should be given to the user concerning the response of the system to any action performed. Better feedback can, for example, eliminate mode errors. Mode errors are common mistakes. They arise when we perform an action appropriate for one mode, but we mistakenly use it for another (e.g. a nurse assumes that the analgesia dispenser is set to a default concentration of $1 \mathrm{mg}$, but in reality it was set to $10 \mathrm{mg}$ by a previous user) [5].

\section{HIS Quality-in-Use Model}

The objective of this section is to present the Quality-in-Use model tailored specifically to HIS user needs. The Goal Question Metric (GQM) approach [1] was applied to define the user needs for the HIS Quality-in-Use goals, and how these goals are further decomposed into more technical sub goals and the corresponding indicators based on HCI principles. 
GQM. The GQM approach is based on the fact that, for an organization to measure in a purposeful way, it must first specify goals for itself and its projects, then it must trace those goals to the data that are intended to define them operationally, and, finally, it must provide a framework for interpreting the data with respect to those goals [1]. The result of applying the GQM approach is a description of a measurement model targeting a particular set of issues and a set of rules for the interpretation of the measurement data. A GQM model is a hierarchical structure (Figure 1) which starts with a goal. That goal is refined into several sub goals using appropriate questions which usually break the issue down into its quantitative or qualitative indicators. Each indicator is then refined into a measurement procedure and rules for data interpretation.

Quality-in-Use. Quality-in-use is the user's view of the quality of an environment containing software and is a broader view than software usability. It is measured based on the results of using the software in the environment (here, the medical field), rather than on properties of the software itself [14]. The ISO/IEC 9126-4 International Standard describes a two-part model for software quality: a) internal quality and external quality, and b) quality-in-use. Quality-in-use is defined as the capability of the software product to enable specified users to achieve specified goals with effectiveness, productivity, safety, and satisfaction in specified contexts of use [14]. In ISO/IEC 9126-4, effectiveness is defined as the capability of the software product to enable users to achieve specified goals with accuracy and completeness; productivity is defined as the capability of the software product to enable users to expend appropriate amounts of resources, such as time, to complete the task or the user's effort, in relation to the effectiveness achieved; safety is described as the capability of the software product to achieve acceptable levels of risk of harm to people, businesses, software, property, or the environment; and, finally, satisfaction is expressed in terms of the user's response to interaction with the product, which includes attitudes towards the use of the product).

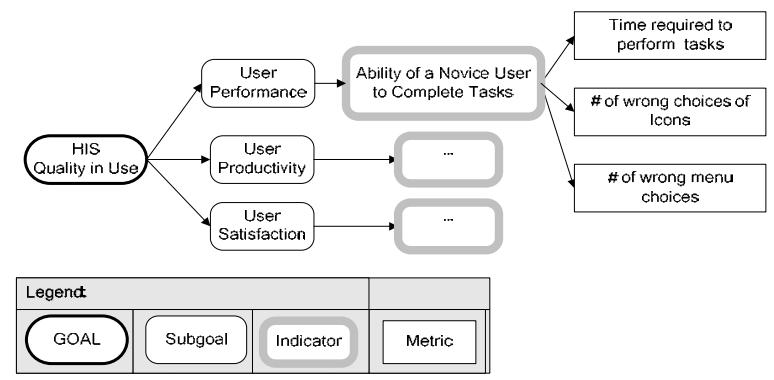

Fig. 1. Hierarchical Quality-in-Use model for HIS (User Performance subgoal)

HIS Quality-in-Use. The quality-in-use model proposed in this research is rooted in the ISO/IEC 9126-4 definition of quality-in-use [14] and is aimed at helping medical personnel execute work-related tasks, in terms of: i) reducing the medical errors by making the use of HIS more pleasant and easier to manipulate, and ii) increasing their performance, productivity and satisfaction by means of HCI principles. The GQM approach was applied to further decompose user performance, user productivity, and user 
satisfaction sub goals into their corresponding indicators providing feedback on the achievement of the user sub goals, and measurement procedures for obtaining the data required for the feedback. The decomposition is motivated by the HCI principles in the specific context of use, namely, HIS in the medical field. Figures 1, 2, and 3 illustrate the above decomposition for user performance, productivity, and user satisfaction.

Validation of the HIS Quality-in-Use model proposed in this paper consisted of demonstrating the improvement of quality-in-use characteristics user performance, productivity, and satisfaction on existing software, The Phoenix Health Information

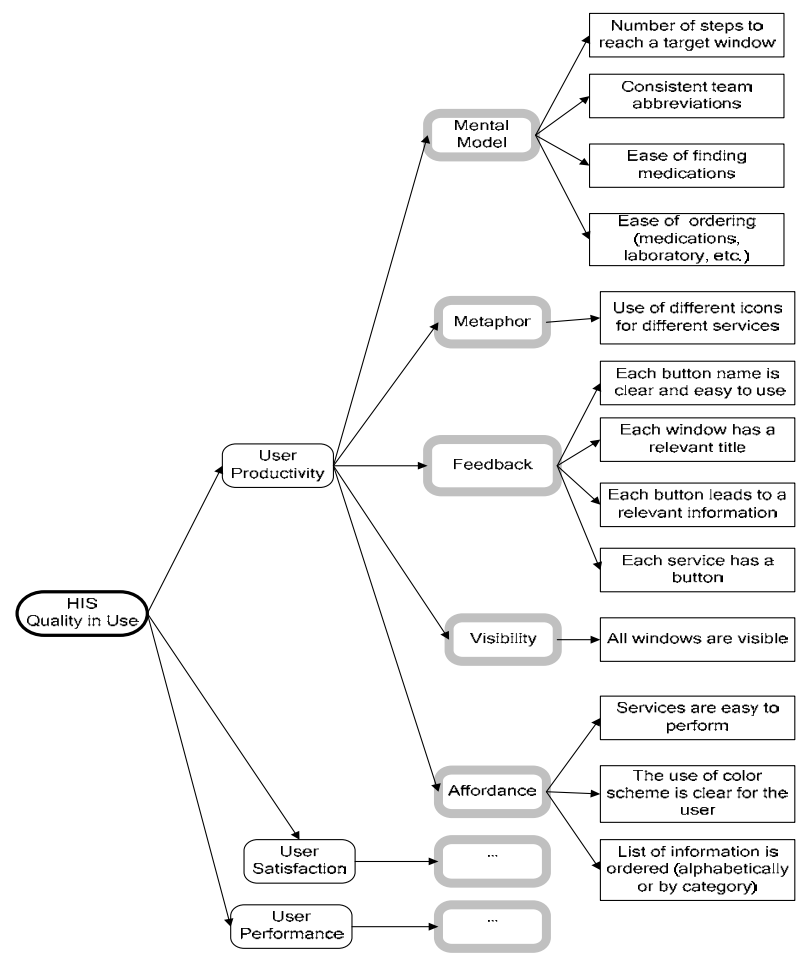

Fig. 2. Hierarchical Quality-in-Use model for HIS (Productivity subgoal)

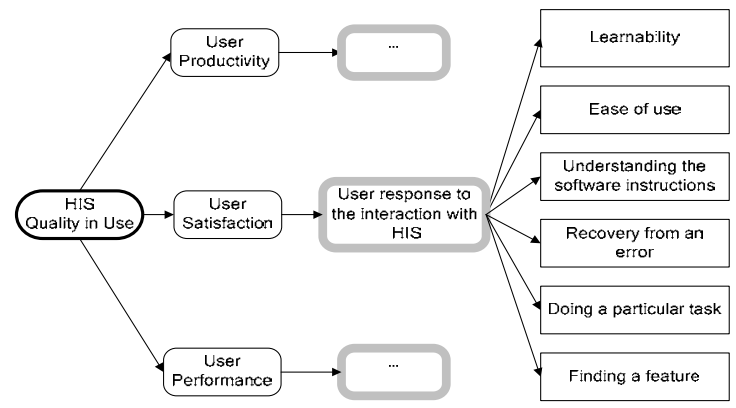

Fig. 3. Hierarchical Quality-in-Use model for HIS (User Satisfaction subgoal) 
System (PHIS, see section 5.1), which, after applying the HCI principles, produced a new version of the system (PHIS2, see section 5.2).

\section{Case Study}

An example of medical software is the Phoenix Health Information System (PHIS). The creation of the PHIS began in 1998 after a team from New Zealand and other countries met in Dubai to evaluate current health information systems. King Abdulaziz University Hospital (KAUH) started to use the PHIS in 2004 to replace the Oasis system. The quality-in-use quality criteria were formally evaluated on the PHIS by novice and expert users from the hospital to identify problems they were having with the system. The researcher concluded that all the problems found based on the tasks doctors commonly perform on a daily basis were rooted in a violation of the HCI principles Mental Model, Metaphor, Visibility, Affordance, and Feedback.

\subsection{PHIS}

Identifying and selecting users. The PHIS is divided into three parts. Each part serves a different group of people in the hospital: administrators, nurses, and doctors. This study focuses on the PHIS subsystem used by doctors and the tasks they commonly carry out on a daily basis. The doctors at the KAUH are listed in three categories: interns, residents, and consultants. Interns use the system the most frequently. The characteristics of the interns are summarized in Table 1.

Table 1. Characteristic of the Interns

\begin{tabular}{llc}
\hline \multicolumn{1}{c}{ Type of Intern } & Experienced & Novice \\
\hline Work & \multicolumn{1}{c}{ In various wards } \\
$\begin{array}{l}\text { Place } \\
\text { Time }\end{array}$ & \multicolumn{1}{c}{ FAUH } \\
Using the PHIS & From 3 to 7 months AM to 5:00 PM \\
Training Time & Two hour training session available for all new doctors \\
Attended the training session & $80 \%$ & From 3 to 5 days \\
\hline
\end{tabular}

Collecting data and identifying problems. The two data collection methods used to gather information from experienced and novice users were interviews and observations. Responses and feedback from both groups were recorded:

Experienced users: Ten experienced PHIS users were interviewed and observed. These interviews provided the researchers with a clear picture of the problems users encountered regarding learnability and ease of use. The interviews also identified the daily tasks most commonly performed by the doctors on the system, such as: i) review the result of a laboratory or radiology test and check a patient's prescribed medication, ii) request a new test, and iii) book an appointment for an operation.

Novice users: Ten novice PHIS users were interviewed and observed while they carried out a list of tasks on the PHIS to evaluate their ability to use the system without help (Table 2). 
Table 2. Results of the Task Questionnaire

\begin{tabular}{lccccc}
\hline & $\begin{array}{c}\text { Task1 Check } \\
\text { a laboratory } \\
\text { result }\end{array}$ & $\begin{array}{c}\text { Task2 Print a } \\
\text { laboratory } \\
\text { result }\end{array}$ & $\begin{array}{c}\text { Task3 Request } \\
\text { an order for } \\
\text { radiology }\end{array}$ & $\begin{array}{c}\text { Task4 Book an } \\
\text { appointment for an } \\
\text { operation }\end{array}$ & $\begin{array}{c}\text { Task5 Collect } \\
\text { blood sample }\end{array}$ \\
\hline User 1 & $\times$ & $\times$ & $\times$ & $\times$ & $\times$ \\
User 2 & $\checkmark$ & $\times$ & $\checkmark$ & $\times$ & $\checkmark$ \\
User 3 & $\times$ & $\times$ & $\checkmark$ & $\times$ & $\checkmark$ \\
User 4 & $\times$ & $\times$ & $\checkmark$ & $\times$ & $\times$ \\
User 5 & $\times$ & $\times$ & $\times$ & $\times$ & $\times$ \\
User 6 & $\checkmark$ & $\checkmark$ & $\times$ & $\times$ & $\times$ \\
User 7 & $\checkmark$ & $\times$ & $\times$ & $\times$ & $\checkmark$ \\
User 8 & $\checkmark$ & $\times$ & $\checkmark$ & $\times$ & $\times$ \\
User 9 & $\times$ & $\times$ & $\times$ & $\times$ & $\times$ \\
User 10 & $\times$ & $\times$ & $\times$ & $0 / 10$ & $4 / 10$ \\
\hline Total & $4 / 10$ & $1 / 10$ & $4 / 10$ & & $\times$ \\
\hline
\end{tabular}

All the interviewees were found to be competent computer users. Five tasks were presented to the users in the questionnaire (Table 2): a check $(\checkmark)$ means that the user performed the task successfully, while a cross $(\times)$ means that the user was unable to finish the task or to start the task. Forty percent of the users were able to complete tasks 1, 3, and 5, while the remaining users were no. Only $10 \%$ could complete task 2 and none of the users was able to complete task 4. Even though the users were proficient in terms of their computer skills and attended the training session, fewer than $50 \%$ were able to complete the tasks given to them.

Table 3 lists the HIS tasks most commonly performed by the doctors, and the applicable HCI principles:

Table 3. Tasks Given to the Novice Group

\begin{tabular}{|c|c|}
\hline Tasks & HCI Principles \\
\hline Task 1.1 Check the last HB & Mental Model \\
\hline Task 1.2 Print the result & Metaphor \\
\hline Task 1.3 Compare CBC Tests? & Metaphor \\
\hline Task 1.4 Print HB from Compare Item & Mental Model and Visibility \\
\hline Task 2 Order CBC & Visibility and Feedback \\
\hline Booking & Affordance \\
\hline Task 4 Check the radiology result & Mental Model, Visibility, and Feedback \\
\hline Task 5 Print medication report & Mental Model and Feedback \\
\hline
\end{tabular}

Summary of HCI Problems in the PHIS. The results of interviewing the ten experienced users and the ten novice users indicated that the PHIS is not user-friendly and not easy to learn, and there are several interface design faults which need attention. The proposed quality indicators helped reveal the existing PHIS HCI problems, as outlined below (the problems are grouped by indicator for clarity):

\section{Mental Model}

- Lengthy steps required to reach a target window

- Selecting a ward does not filter the teams

- Team abbreviations are inconsistent from one ward to another 


\section{Metaphor}

- Use of the same icon for different functions, e.g. $\hat{\text { P Profile }} \mid$ and 2 Patient List..

\section{Feedback}

- Function name of the window title displayed, instead of the window title

- In the Profile tab:

- there is a list of buttons using vague abbreviations (e.g. MAR/IVAR)

- patient's demographic information is not displayed

\section{Visibility}

- Invisible order window

\section{Affordance}

- Diagnosis list not arranged alphabetically or by category

- Doctors tried to click on a time text box without noticing the background color

The obstacles and problems encountered by the doctors were solved based on the principles of HCI from a design point of view to minimize human error and user confusion, as well as to increase the efficiency and effectiveness. The result was PHIS2, a redesigned and improved version of the PHIS developed through a process that included pilot testing, redesigning, and redeveloping its user interface when it did not reflect HCI principles.

\subsection{PHIS2}

The following illustrates the improvement of the quality-in-use of PHIS2 by comparing the PHIS and PHIS2 quality indicators per sub goal:

User Productivity improvement. After conducting the formal quality assessment of the PHIS and identifying the HCI principles that had been violated, all the recommendations from the findings were applied to improve user productivity with PHIS2. The productivity obstacles and problems encountered by the doctors were solved based on HCI principles from a design point of view to minimize human error caused by the poor quality user interface design of the PHIS.

User Performance improvement. The ability of the user to complete a task successfully is used to evaluate the user's performance. The performance measures are defined in Table 4.

Table 4. Performance Measurement

\begin{tabular}{ll}
\hline Performance measures & Metric \\
\hline Time needed by the novice user to perform task successfully & End Time- Start Time \\
How many times he/she chose the wrong icon & \# of wrong icons selected \\
How many times he/she made the wrong menu choice & \# of wrong menu choices \\
\hline
\end{tabular}

Based on the technique suggested by Dumas and Redish [15], the performance of three experienced users was considered as the baseline for judging the time taken by participants. If their performance was considered excellent, more time was considered acceptable and much more time was considered unacceptable. Table 5 shows an example of the selected performance measures for the 'Check the last HB' task. Each 
Table 5. Setting Criteria for Measuring Performance

\begin{tabular}{lccl}
\hline Measure & Excellent & Acceptable & Unacceptable \\
\hline Task 1.1: Check the last HB & & & \\
Time for task & $<1.5 \mathrm{M}$ & $1.5-2 \mathrm{M}$ & $>2 \mathrm{M}$ \\
M= \# of wrong menu choices & 1 & $2-3$ & More than 3 \\
I= \# of wrong icon choices & 1 & $2-3$ & More than 3 \\
\hline
\end{tabular}

task for each participant was measured and the data compared to that in Table 5, which classifies user performance as excellent, acceptable, and unacceptable. The results show that the performance of the novice group was excellent for more than $80 \%$ of the users for Tasks $1.3,2,4$, and 5, and for $70 \%, 80 \%$, and $60 \%$ of the users for Tasks 1.1, 1.2, and 3 respectively. For Task 1.4, performance was excellent for $50 \%$ of the users and acceptable for $50 \%$ of the users. In only one case was performance unacceptable, that is, for $20 \%$ of the users for Task 1.1 .

The data on the number of wrong menu choices show that performance was excellent for Task 4 and Task 5 for $100 \%$ of the users and for Task 1.1 for $90 \%$ of the users. The data on the number of wrong icons chosen show that performance was excellent for all tasks except Task 3 for $100 \%$ of the users, with performance for Task 3 being excellent for $80 \%$ of the users.

It was interesting to compare the performance of the PHIS with the performance of PHIS2 by ten novice users performing four identical tasks. These tasks represent the Mental Model in Task 1.1, Metaphor in Task 1.2, Feedback and Visibility in Task 2, and Affordance in Task 3. The performance measure in the PHIS was accomplishment of the task, irrespective of the time taken and the number of attempts. Using PHIS2, the novices were able to accomplish their tasks without previous training, while using the PHIS, despite the in-house training, fewer than $40 \%$ were able accomplish their tasks.

From the user performance evaluation, it is clear that the novice users were able to perform all the tasks with PHIS2 in excellent time, which reflects the accuracy of the proposed design. It also shows that their performance with respect to the number of wrong menus choices and icons was also excellent, which reflects the efficiency and effectiveness of the new UI design.

User Satisfaction improvement. The users were asked to complete a User Satisfaction Questionnaire to determine users' impressions and opinions of the system, evaluating the user response to the interaction with PHIS2. The results of the questionnaire revealed that all the users gave ratings of 'very easy' and 'easy' for the tasks regarding learning the program, using the program, performing the particular task, finding the features, understanding the instruction, and recovering from error. In addition, $100 \%$ of the experienced users preferred using PHIS2 to using the PHIS, and 100\% of the participants asserted that PHIS2 helps them improve the quality of their work.

\section{Conclusion and Future Work}

The purpose of this research was the application of HCI principles, based on a sound goal-driven approach, to derive a new quality-in-use model from the medical field users' view of the quality of HIS interfaces which will help to minimize human error and user frustration, and to make the tasks of doctors - with little or no training more pleasant, as well as efficient and effective. Such a model can serve not only to 
evaluate the quality-in-use of an existing HIS, but also as a guide for the user interface design of new medical software.

The PHIS evaluation based on our HIS Quality-in-Use model resulted in major improvements to the user interface design in its improved version, PHIS2. Results of the formal evaluation of PHIS2 reveal that the system is a success in terms of helping users perform tasks in excellent or acceptable time, efficient and effective in terms of limiting the number of wrong menu choices made and wrong icons selected, and more pleasant to use, even with no training whatsoever.

More case studies will be evaluated in our future work to determine the applicability of our quality-in-use model to different types of HIS.

\section{References}

1. Basili, V., Caldiera, G., Rombach, H.D.: The Goal Question Metric Approach (1) Institute for Advanced Computer Studies, Department of Computer Science, University Of Maryland, College Park, Maryland (2) FB Informatik, Universität Kaiserslautern, http: / / wwwagse. informatik.uni-kl.de/pubs/repository/

basilig4b/encyclo.gqm.pdf

2. The Canadian International Development Agency (CIDA) and managed by the Canadian Society for International Health, The South Caucasus Health Information Project (2004), http://www.csih.org/what/schip/March

3. Kushniruk, A.: Evaluation in the design of health information systems: application of approaches emerging from usability engineering. Computers in Biology and Medicine 32, 141-149 (2002)

4. Fabbrini, F., Fusani, M.: Evaluation of Quality Characteristics in Health Care Information Systems, ERCIM News No.28 (January 1997),

http://www.ercim.org/publication/Ercim_News/enw28/

fabbrini.html

5. Burnham. Dr. W.: The Human Factor, http: / /www. humanfactorsmd. com/hfandmedicine.html (2005)

6. Sawyer, D.: An Introduction to Human Factors in Medical Devices, Office of Health and Industry Programs, U.S Department of Health and Human Services, Center for Devices and Radiological Health (1996),

http: / / www . fda.gov/cdrh/humfac/doitpdf .pdf

7. Nielsen, J.: Medical Usability: How to Kill Patients through Bad Design (2005), http://www.useit.com/alertbox/20050411.html

8. Marcus, A.: Metaphor Design in User Interfaces: How to Manage Expectation, Surprise, Comprehension, and Delight Effectively. California (1997), http://www.sigchi . org/sigchi/chi97/proceedings/tutorial/am.htm

9. Dix, A., Finlay, J., Abowd, G., Beale, R.: Human-Computer Interaction, 3rd edn. British Library Cataloguing, England (2004)

10. Booth, P.: An Introduction To Human-Computer Interaction, 4th edn. Lawrence Erlbaum Associates, Mahwah (1989)

11. Norman, D.: The Design of Everyday Things. Doubleday/Currency, New York (1988)

12. McCracken, D., Wolfe, R.: User-Centered Website Development: A Human- Computer Interaction Approach. Pearson Education, USA (2004)

13. Norman, D.: Introduction to HCI, B.Sc. Applied Computing (2004), http://hamilton.bell.ac.uk/btech/hci/hciintro.pdf

14. ISO/IEC 9126-4:2001, Software engineering — Product quality — Part 4: Quality -in-Use

15. Dumas, J.L., Redish, J.C.: A Practical Guide to Usability Testing, 3rd edn. Intellect, Wiltshire (1999) 